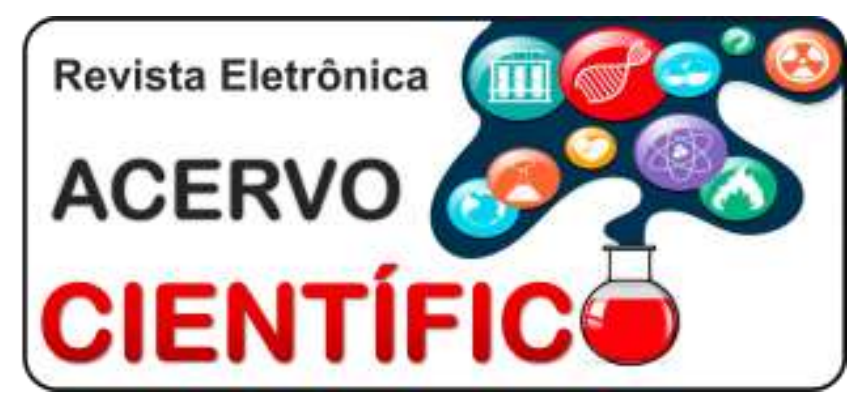

RELATO DE CASO

Recebido em: 11/2019

Aceito em: 11/2019

Publicado em: 11/2019

\title{
Conduta na urgência de fratura peniana com ruptura parcial de uretra: relato de caso
}

Trauma and reconstruction penile fracture with partial urethral rupture: case report Conducta en la urgencia de fractura peniana con ruptura parcial de uretra: relato de caso

Bárbara Oliveira Reis ${ }^{1}$, Denise Junqueira Dos Santos ${ }^{1}$, Flávia Brito De Macedo1, Gislaine Fernandes Gomes ${ }^{1}$, Angélica de Paula Langame ${ }^{1}$, Antônio Carlos Tonelli De Toledo, Fabiano Libório Campos²

Resumo: A fratura peniana é uma situação incomum de ruptura da túnica albugínea com o pênis no estado ereto. Gera significativo constrangimento social e necessita de abordagem adequada. Objetivou-se relatar um caso de fratura com lesão subtotal de uretra abordado cirurgicamente em Juiz de Fora-MG. MAFS, negro, 35 anos, deu entrada no Hospital de Pronto Socorro relatando que durante a atividade sexual ouviu estalido, seguido de jato de sangue, perda da ereção e edema local. Negava dor, parestesias, desvio do órgão, sintomas associados, comorbidades, quadros prévios similares e doenças urogenitais. Foi submetido a tratamento cirúrgico de urgência, onde se confirmou o diagnóstico. Evoluiu bem e permaneceu com sondagem vesical por catorze dias. Os métodos de imagem foram desnecessários neste caso, mas podem auxiliar no diagnóstico, quando existirem aspectos confundidores. O manejo cirúrgico urgente foi associado a boa recuperação. Acredita-se que exames complementares são dispensáveis quando indisponíveis, se houver poucos recursos e se a anamnese e o exame físico forem altamente sugestivos, pois o diagnóstico da fratura peniana é essencialmente clínico e, além disso, a intervenção cirúrgica no tratamento desse quadro juntamente com lesão uretral deve ser feita precocemente para restabelecimento da função urinária, erétil e prevenção de sequelas.

Palavras-chave: Pênis, Ruptura, Gerenciamento Clínico.

Abstract: Penile fracture is an unusual condition of rupture of the albuginous tunic with the penis in an erect state. It generates significant social embarrassment and needs proper approach. This study aimed to report a case of fracture with subtotal lesion of the urethra surgically approached in Juiz de Fora-MG. MAFS, a 35year-old black man, was admitted to the Emergency Room Hospital reporting that during sexual activity he heard a crackling sound, followed by blood jetting, loss of erection and local edema. He denied pain, paresthesias, organ deviation, associated symptoms, comorbidities, similar previous conditions and

\footnotetext{
${ }^{1}$ Hospital Universitário da Universidade Federal de Juiz de Fora, (HU-UFJF), Juiz de Fora-MG.

E-mail: barbarareisjf@gmail.com

${ }^{2}$ Hospital de Pronto Socorro Dr. Mozart Teixeira (HPS), Juiz de Fora-MG.
} 
urogenital diseases. He underwent urgent surgical treatment, which confirmed the diagnosis. She evolved well and remained with bladder catheterization for fourteen days. Imaging methods were unnecessary in this case but may aid in diagnosis when confounding aspects exist. Urgent surgical management was associated with good recovery. Complementary exams are believed to be unnecessary when unavailable, if resources are scarce and if anamnesis and physical examination are highly suggestive, since the diagnosis of penile fracture is essentially clinical and, in addition, surgical intervention in the treatment of this condition together with Urethral injury should be done early for restoration of urinary, erectile function and prevention of sequelae.

Keywords: Penis, Rupture, Clinical Management.

Resumen: La fractura de pene es una condición inusual de ruptura de la túnica albuginosa con el pene en estado erecto. Genera una vergüenza social significativa y necesita un enfoque adecuado. Este estudio tuvo como objetivo informar un caso de fractura con lesión subtotal de la uretra abordada quirúrgicamente en Juiz de Fora-MG. MAFS, un hombre negro de 35 años ingresó en el Hospital de la sala de emergencias informando que durante la actividad sexual escuchó un crujido, seguido de chorro de sangre, pérdida de la erección y edema local. Negó dolor, parestesias, desviación de órganos, síntomas asociados, comorbilidades, afecciones previas similares y enfermedades urogenitales. Se sometió a un tratamiento quirúrgico urgente, que confirmó el diagnóstico. Ella evolucionó bien y permaneció con cateterismo vesical durante catorce días. Los métodos de imagen fueron innecesarios en este caso, pero pueden ayudar en el diagnóstico cuando existen aspectos confusos. El manejo quirúrgico urgente se asoció con una buena recuperación. Se cree que los exámenes complementarios son innecesarios cuando no están disponibles, si los recursos son escasos y si la anamnesis y el examen físico son altamente sugestivos, ya que el diagnóstico de fractura de pene es esencialmente clínico y, además, la intervención quirúrgica en el tratamiento de esta afección junto con La lesión uretral debe realizarse temprano para restaurar la función urinaria y eréctil y prevenir las secuelas.

Palabras clave: Pene, Ruptura, Manejo clínico.

\section{INTRODUÇÃO}

A fratura peniana representa ruptura da túnica albugínea com o pênis no estado ereto. Gera significativo constrangimento social e necessita de abordagem adequada. Apesar do nome, não se trata de uma fratura propriamente dita, visto que o pênis não é um órgão ósseo. Um atraso na intervenção gera perda significativa em anos de capacidade sexual. É uma situação incomum com incidência de 1 em 175 pessoas e idade média de 28 anos (AMER T, 2016).

Essa lesão ocorre, na maioria das vezes, quando o pênis é golpeado contra o períneo no intercurso sexual ou durante a masturbação (CASTRO PR, 2009). Sendo a túnica albugínea uma das fáscias mais fortes do corpo humano, o risco de fratura peniana aumenta durante a ereção, devido à pressão sob sua estrutura e pelo seu estiramento e afinamento, reduzindo sua espessura em 75\% (CARVALHO J, 2013; ANDRADE MF, 2015).

O diagnóstico de fratura de pênis é baseado na história clínica e exame físico, devendo ser suspeitado quando o paciente referir súbito estalido durante atividade sexual seguido de edema e descoloração peniana, que caracterizam a deformidade conhecida como "sinal da berinjela" (CASTRO PR, 2009; MAHAPATRA RS, et al., 2015).

De acordo com a European Association of Urology (EAU), se houver incerteza diagnóstica, pode-se lançar mão de métodos de imagem como: ultrassonografia, ressonância magnética e cavernosografia, os quais localizarão detalhadamente a lesão. O ultrassom é menos invasivo, porém examinador-dependente e de visualização prejudicada pelo edema e hematoma, perdendo neste sentido para Ressonância, que é 
menos acessível. A carvenosonografia pode fornecer resultados falso-negativos, demandar longo tempo para realização e gerar complicações, como reações alérgicas devido ao contraste infundido. Juntamente com a fratura de pênis, há 10 a $20 \%$ de chances de ocorrer ruptura uretral e esse quadro deve ser suspeitado quando houver uretrorragia, hematúria macroscópica, sintomas miccionais e retenção urinária, podendo ser confirmado com a uretrocistografia retrógrada e cistoscopia flexível, exames de maior acurácia (ALVES LS, 2004; CARVALHO J, 2013; DJAKOVIC N, et al., 2005; GAROFALO M, et al., 2015).

O reparo cirúrgico é o melhor método para o tratamento da fratura de pênis e reparação da uretra, caso seja confirmada a concomitância das duas lesões, quando comparado ao tratamento conservador através de imobilização compressiva, medicações fibrinolíticas, antibióticos e inibidores da ereção (GAROFALO M, et al., 2015).

O tipo de cirurgia depende da experiência e escolha do cirurgião, podendo ser uma incisão peniana por circuncisão com desenluvamento da superfície peniana ou uma incisão longitudinal sobre a área esperada do defeito, com posterior sutura dos locais apresentando perda de continuidade tecidual (ORY J, 2019). As recomendações incluem abstinência sexual por aproximadamente 6 semanas.

O objetivo deste artigo foi relatar um caso de fratura de pênis associado à injúria uretral e seguido de tratamento cirúrgico.

\section{DETALHAMENTO DO CASO}

MAFS, masculino, negro, 35 anos, entregador, em união estável, natural de Guapimirim-RJ, residente em Juiz de Fora - MG, deu entrada no dia 10 de setembro às 10 horas em um hospital de urgência relatando que, no intercurso sexual com a companheira há aproximadamente 1 hora, ouviu estalido juntamente com eliminação de jato de sangue pelo meato uretral externo e perda da ereção. Em seguida, apresentou edema local com uretrorragia intensa.

Ao ser examinado, foi diagnosticada fratura de corpo cavernoso, caracterizando uma urgência urológica. Negava dor, parestesias, deformação e curvatura peniana visível, sintomas associados, uso de medicações, comorbidades, quadros prévios similares e doenças urogenitais.

Após avaliação clínica, foi acionado urologista de plantão para conduta cirúrgica. Ao ser levado para o centro cirúrgico, foi submetido a anestesia raquidiana e sedado sendo a operação iniciada por médicos Associados a Faculdade de Medicina da Universidade Federal de Juiz de Fora, os quais identificaram rotura da túnica albugínea. Primeiramente, foi realizada postectomia e desenluvamento peniano. Durante a cirurgia, detectou-se ruptura parcial da uretra peniana e fratura dos corpos cavernosos. A uretra foi reparada com cateterização e os corpos cavernosos reconstruídos.

O paciente em questão manteve-se em repouso nos 2 primeiros dias do pós-operatório, durante os quais manteve-se com uretrorragia, tendo a sonda vesical de demora permanecido por 14 dias. Em visita médica no dia seguinte ao procedimento, relatou dor nas regiões suturadas e à mobilização peniana, com significativa redução do edema e sensibilidade tátil aumentada pela exposição da glande. Negava febre. À inspeção, os pontos se apresentavam íntegros, a ferida cirúrgica limpa e a sonda em perfeito funcionamento, sendo realizado novo curativo. Recebeu alta hospitalar após 5 dias, sendo orientado a permanecer em repouso, evitar relação sexual por 3 meses e realizar higienização.

Relatou febre não aferida no primeiro dia de alta, obtendo melhora através do uso de Dipirona. Foi retirada a sonda vesical no 14ํㅡㄹ dia do pós-operatório, em consulta de revisão com o cirurgião responsável. A área abordada cirurgicamente apresentava-se sem sinais flogísticos e há 4 dias havia cessado a queixa de sensibilidade aumentada na região postectomizada. Neste momento, o paciente apresentava dor suprapúbica associada à sondagem vesical, e relatou ter tido ereção sem deformidades penianas visíveis no pós-operatório.

Ao final da consulta, MAFS assinou Termo de Consentimento Livre e Esclarecido e permitiu entrevista para coleta detalhada dos dados da anamnese. Solicitou-se a realização de um exame de urina após 6 dias 
da retirada da sonda, para análise dos Elementos Anormais do Sedimento, piúria e hematúria quantitativas, além da urocultura.

\section{DISCUSSÃO}

A confirmação da fratura peniana é feita prioritariamente pela história clínica e exame físico, corroborando com o que foi feito no caso clínico (CASTRO PR, 2009; CARVALHO J, 2013; ALVES LS, 2004). Caso haja incerteza no diagnóstico, pode-se lançar mão de métodos como a ultrassonografia, ressonância magnética e a cavernografia, sendo que a infusão de soro fisiológico no corpo cavernoso permite a localização precisa da fratura durante o procedimento cirúrgico (GAROFALO M, et al. 2015; ALVES LS, 2004). Porém, conforme pode ser observado no caso, os exames complementares são dispensáveis quando indisponíveis, se houver poucos recursos e se a anamnese e o exame físico forem altamente sugestivos. Portanto, sua ausência não é prejudicial e não deve atrasar a conduta cirúrgica e 0 diagnóstico, gerando questionamentos sobre os atuais protocolos de fratura peniana (SABHARWAL S, et al., 2015).

Na América do Norte é mais comumente associada a relação sexual. Normalmente, o caso típico de fratura acontece quando a mulher está em cima do homem durante o sexo e o pênis sai da cavidade vaginal. Ao tentar penetrar novamente, se choca com o quadril ou períneo da parceira, fazendo com que se dobre de maneira brusca e forçada. Em posições sexuais em que o homem fica acima ou atrás da parceira, os riscos de fratura diminuem consideravelmente. Em países do Oriente Médio, uma causa comum de fratura peniana é a automanipulação (Taghaandan) quando o pênis ereto é dobrado, ou para alcançar rápida detumescência (CARLOS SS, et al., 2013).

Uma compreensão da anatomia peniana, especialmente da complexidade de seus planos fasciais, é essencial para avaliar com precisão a gravidade da fratura peniana, pois essas camadas influenciam o padrão de extravasamento de hematoma após o trauma. As interfaces fasciais do pênis podem ser categorizadas em três camadas distintas - tecido conjuntivo fibroso, facial profundo e superficial. A camada fibrosa chamada túnica albugínea é uma bainha fibro-elástica densa que envolve ambos corpos cavernosos e é fundida na linha média entre eles. Essa camada incorpora um estrato composto por fibras de orientação longitudinal que se ligam aos ramos pubianos e uma camada circular que contribui para formar o septo entre as cavernas. É considerada a fáscia mais resistente do corpo e a mais importante na definição de uma fratura peniana (NAPIER D, 2019). Na maioria das vezes, a fratura ocorre na região proximal da haste peniana. A laceração da albugínea tipicamente é unilateral e transversa, localizando-se na porção ventral, distal ao ligamento suspensório do pênis (ALVES LS, 2004).

Em 2015, Shukla AK, et al (2015), compuseram um sistema de classificação de ruptura peniana com base nas aparências ultrassonográficas mais comuns encontradas em sua experiência clínica. Esse sistema de classificação identificou quatro subtipos principais, variando de 0 a 4 (NAPIER D, 2019). O paciente do caso apresentou grau 4 de gravidade de lesão peniana, a qual é caracterizada por lesão de corpo cavernoso ou defeito uretral maior que $2 \mathrm{~cm}$ - penectomia parcial. Quanto à lesão uretral sugerida pela presença de hematúria e uretrorragia, a mesma pode ser investigada pela uretrocistografia retrógrada e uretroscopia. Neste caso, lesão da uretra do paciente era de gravidade grau 3, caracterizada por ruptura parcial e extravasamento do contraste no local e na bexiga (DJAKOVIC N, et al., 2005).

O primeiro manejo cirúrgico da fratura peniana foi descrito em 1936 por Fetter e Gartman e desde então, este meio de reparo é o padrão-ouro para o tratamento deste quadro (MAHAPATRA RS, et al., 2015; GAROFALO M, et al., 2015). A abordagem cirúrgica está associada à menor duração da internação hospitalar, menores custos, maiores níveis de satisfação do paciente e melhores resultados quanto a capacidade mictória e redução da incidência de disfunção erétil (GAROFALO M, et al., 2015). O tratamento cirúrgico consiste na drenagem do hematoma, controle da hemorragia, sutura dos corpos cavernosos e da uretra, caso esta esteja lesada (ANDRADE MF, 2015). Opta-se pelo tratamento conservador apenas em situações especiais como: objeção do paciente à cirurgia; apresentação atrasada da lesão ao serviço de 
saúde devido ao constrangimento; fraturas e hematomas pequenos ou rupturas mínimas dos corpos cavernosos (DJAKOVIC N, et al., 2005; ALVES LS, 2004; MUENTENER M, et al., 2004).

Os resultados mais promissores relacionam-se também com a abordagem mais precoce nas primeiras 24 horas em relação às tardias (CARVALHO J, 2013; GAROFALO M, et al., 2015). Estudo mostrou que pacientes submetidos ao tratamento cirúrgico tardio podem evoluir com sequelas, como: curvatura homolateral à lesão, diminuição da qualidade da ereção e formação de calo ósseo (MUENTENER M, et al., 2004). Segundo Carlos SS, et al (2013), o tratamento cirúrgico imediato pode levar a taxas de curvatura peniana em índices menores que 5\%, enquanto o tratamento conservador apresenta taxas maiores que $10 \%$, além da possibilidade de apresentarem abscessos ou placas de fibrose debilitantes em 25 a $30 \%$. Além disso, a intervenção imediata tem sido associada a menor tempo de hospitalização, níveis mais altos de satisfação do paciente e a melhores resultados, incluindo reduzida incidência de disfunção erétil, sendo a função eréctil normal, na ausência de curvatura ou cicatriz, observada em $77 \%$ dos pacientes.

Após a cirurgia recomenda-se a utilização de curativo compressivo e, em alguns casos, cateterismo vesical de demora. Sobre a utilização e o tempo de sondagem uretral, os estudos atuais não demonstram consenso, deixando à cargo do cirurgião a escolha de seu uso (IBARGUREN RL, et al., 2006). Também não há consenso sobre a necessidade de supressão química da ereção no pós-operatório e, apesar de ter sido feita em alguns estudos, não houve confirmação de grau de evidência que sugira seu uso rotineiro (MAHAPATRA RS, et al., 2015; FERNANDES MAV, et al., 2018; HOAG NA, et al., 2011; KOIFMAN L, et al., 2003; MOREY AF, 2007).

Os fatores de risco para fraturas recorrentes envolvem evidências histológicas de um processo inflamatório crônico subjacente na túnica albugínea de pacientes com fratura peniana, em que tecido cicatricial fibroso e inelástico da lesão anterior enfraqueceria o corpo cavernoso, tornando-o mais fraco e vulnerável a um novo episódio de fratura. Essa teoria é corroborada pela predominância de casos de fratura que se repetem no corpo cavernoso ipsilateral. Porém também, há evidências de que o tecido cicatricial poderia levar a uma distribuição desigual de tensão na túnica albugínea, causando ruptura do lado contralateral (BARROS R, et al., 2018).

\section{CONSIDERAÇÕES FINAIS}

A fratura peniana deve ser avaliada adequadamente, principalmente pela possibilidade de associação à lesão uretral e pelo risco de sequelas, buscando, na história clínica e no exame físico, dados para o diagnóstico. Os sinais mais frequentes são edema e descoloração. Já o sangramento uretral, os sintomas miccionais e a retenção urinária são sinais indicativos de ruptura uretral. Os métodos de imagem podem auxiliar no diagnóstico se existir aspectos confundidores, porém, conforme pode ser observado nesse caso, são dispensáveis quando ausentes e se a clínica e o exame físico forem altamente sugestivos. Como terapêutica, consagra-se o manejo cirúrgico precoce associado à melhores resultados. Embora seja uma emergência urológica rara, tem um impacto significativo na visão geral e na saúde sexual. Sendo a idade média da lesão de 28 anos, um atraso na intervenção gera perda significativa em anos de capacidade sexual. Portanto, a conscientização e a importância de procurar tratamento precoce devem ser explicadas aos profissionais de saúde e a população jovem.

\section{REFERÊNCIAS}

1. ALVES LS. Fratura de Pênis. Revista do Colégio Brasileiro de Cirurgia. 2004; 31(5): 284-286.

2. AMER T. Penile Fracture: A Meta-Analysis. Urologia Internationalis. 2016; 96(3): 315-329.

3. ANDRADE MF, et al. Abordagem Tardia do Trauma de Pênis: Relato de Caso. Revista Ciências em Saúde. 2015; 5 (2).

4. BARROS R, et al. Penile refracture: a preliminary report. International braz j urol. 2018; 44(4), 800-804. 
5. CARLOS SS, et al. Fratura peniana - Uma revisão. Revista Eletrônica da Comissão de Ensino e Treinamento da SBU. 2013 (1)2: 15-20.

6. CARVALHO J, ARLINDO M. Fratura de Pênis com Trauma de Uretra. Revista do Colégio Brasileiro de Cirurgiões. 2013; 40(4): 351-353.

7. CASTRO PR. Fratura Peniana: Diagnóstico e Tratamento. Revista Médica de Minas Gerais. 2009; 19(2):123-126.

8. DJAKOVIC N, et al. Diretrizes para o Trauma Urológico. European Association of Urology. 2005; 47(1): 1-15.

9. FERNANDES MAV, et al. Avaliação ultrassonográfica do pênis. Radiol Bras [Internet]. 2018 Aug [cited 2019 Mar 17] ; 51( 4 ): 257-261.

10. GAROFALO M, et al. Sex-Related Penile Fracture with Complete Urethral Rupture: A Case Report and Review of The Literature. Archivio Italiano di Urologia e Andrologia. 2015; 87(3):260-1.

11. HOAG NA, et al. Penile fracture with bilateral corporeal rupture and complete urethral disruption: case report and literature review. Canadian Urological Association 2011; 5 (2) E23-6.

12. IBARGUREN RL, et al. Penile Fracture with Associated Urethral Rupture. Archivos Espanoles de Urologia. 2006; 59 (7):732-736.

13. KOIFMAN L, et al. Penile fracture-Experience in 56 cases. Int Braz J Urol. 2003; 29:35-9.

14. MAHAPATRA RS, et al. Penile Fracture: Our Experience in a Tertiary Care Hospital. The World Journal of Men's Health. 2015; 33 (2): 95-102.

15. MOREY AF, ROZANSKI TA. Genital and lower urinary tract trauma. Campbell-Walsh Urology. $2007 ; 9$ (3) 83.

16. MUENTENER $\mathrm{M}$, et al. Long-Term Experience with Surgical and Conservative Treatment of Penile Fracture. The Journal of Urology. 2004;172:576-9.

17. NAPIER D. The role of ultrasound in the diagnosis of penile fracture. Sonography. 2019; $6: 15$-23.

18. ORY J, BAILLY G. Management of penile fracture. Can Urol Assoc J. 2019;13 (6) 4:72-74.

19. SABHARWAL S, et al. Hidden Penile Fracture: An Unusual Presentation and Review of Literature. Urology Annals. 2015; 7 (2): 248.

20. SHUKLA AK, et al. Role of ultrasonography in grading of penile fractures. J Clin Diagn Res. 2015; 9(4): $1-3$. 